\title{
A 1-month-old infant with chylomicronemia due to GPIHBP1 gene mutation treated by plasmapheresis
}

\author{
Mo Kyung Jung, $M D^{1}$, \\ Juhyun Jin, MD', \\ Hyun Ok Kim, MD², \\ Ahreum Kwon, MD', \\ Hyun Wook Chae, MD', \\ Seok Jin Kang, MD', \\ Duk Hee Kim, MD, PhD ${ }^{3}$, \\ Ho-Seong Kim, MD, PhD
}

'Department of Pediatrics, Severance Children's Hospital, Yonsei University College of Medicine, Seoul, ${ }^{2}$ Department of Laboratory Medicine, Yonsei University College of Medicine, Seoul, ${ }^{3}$ Sowha Children's Hospital, Seoul, Korea
Received: 30 September, 2016 Revised: 22 October, 2016 Accepted: 15 December, 2016

Address for correspondence: Ho-Seong Kim, MD, PhD

Department of Pediatrics, Severance Children's Hospital, Endocrine Research Institute, Yonsei University College of Medicine, 50-1 Yonsei-ro, Seodaemun-gu, Seoul 03722, Korea Tel: $+82-2-2228-2050$

Fax: +82-2-393-9118

E-mail:kimho@yuhs.ac

https://orcid.org/0000-0003-1135099X
Chylomicronemia is a severe type of hypertriglyceridemia characterized by chylomicron accumulation that arises from a genetic defect in intravascular lipolysis. It requires urgent and proper management, because serious cases can be accompanied by pancreatic necrosis or persistent multiple organ failure. We present the case of a 1-month-old infant with chylomicronemia treated by plasmapheresis. His chylomicronemia was discovered incidentally when lactescent plasma was noticed during routine blood sampling during a hospital admission for fever and irritability. Laboratory investigation revealed marked triglyceridemia $(>5,000 \mathrm{mg} / \mathrm{dL}$ ) with high chylomicron levels. We therefore decided to perform a therapeutic plasmapheresis to prevent acute pancreatitis. Sequence analysis revealed a homozygous novel mutation in exon 4 of GPIHBP1: c.476delG (p.Gly159Alafs). Glycosylphosphatidylinositol-anchored high density lipoproteinbinding protein 1 (GPIHBP1) stabilizes the binding of chylomicrons near lipoprotein lipase and supports lipolysis. Mutations of GPIHBP1, the most recently discovered gene, can lead to severe hyperlipidemia and are known to make up only $2 \%$ of the monogenic mutations associated with chylomicronemia. The patient maintains mild hypertriglyceridemia without rebound after single plasmapheresis and maintenance fibrate medication so far. Here, we report an infant with chylomicronemia due to GPIHBP1 mutation, successfully treated by plasmapheresis.

Keywords: Chylomicronemia, GPIHBP1, Plasmapheresis

\section{Introduction}

Severe hypertriglyceridemia is diagnosed when the plasma triglyceride (TG) concentration exceeds $885 \mathrm{mg} / \mathrm{dL}^{1)}$. Chylomicronemia, formerly known as type 1 hyperlipoproteinemia, is a type of severe hypertriglyceridemia characterized by the accumulation of chylomicrons in fasting plasma ${ }^{2)}$.

Chylomicronemia usually presents in infancy or childhood with episodes of eruptive xanthomas on the trunk and extremities, lipaemia retinalis, recurrent abdominal pain, acute and/or chronic pancreatitis and hepatosplenomegaly ${ }^{3)}$. It is often discovered incidentally by lactescent plasma during routine blood sampling. The most serious manifestation is pancreatitis, which is associated with $5 \%-6 \%$ overall mortality, and the highest mortality rate is related to the development of pancreatic necrosis or persistent multiple organ failure ${ }^{4)}$.

Familial chylomicronemia has a monogenic basis with autosomal recessive pattern and arises from inactivating mutations in genes that encode key check-point molecules in lipolysis such as lipoprotein lipase (LPL), apolipoprotein C2 (APOC2), apolipoprotein A5 (APOA5), lipase maturation factor 1 (LMF1), or glycosylphosphatidylinositol-anchored high density lipoprotein-binding protein 1 (GPIHBP1 $)^{5,6)}$.

GPIHBP1 binds LPL and translocates the enzyme to the luminal surface of capillary endothelial cells, thus supporting lipolysis ${ }^{7)}$. GPIHBP1 mutations may disturb lipolytic 
processing, which leads to severe hypertriglyceridemia, and to date this disturbance has been reported in only 26 families $^{8-11)}$.

The mainstay of treatment is a restriction of fat intake to less than or equal to $15 \%$ of total energy intake, and especially infants are given medium chain triglycerides (MCTs) milk, which are not incorporated into chylomicrons ${ }^{12)}$. However, in cases with very high TG levels, dietary treatment may be insufficient for preventing pancreatitis, and nonpharmacological treatment, such as plasma exchange, becomes necessary.

Here, we report a 1-month-old infant with chylomicronemia due to a GPIHBP1 mutation and how he was treated by extracorporeal methods.

\section{Case report}

A 40-day-old male infant, born at 39 weeks and $3.3 \mathrm{~kg}$ by caesarean section without perinatal complications, was hospitalized with fever and irritability for 1 day. His height was $56 \mathrm{~cm}$ (25th-50th percentile) and weight was $4.6 \mathrm{~kg}$ (5th-10th percentile) with absolute breastfeeding. When a blood sample was taken for evaluation, physicians noticed lactescent plasma. He was then referred to the pediatric endocrinology clinic. He was the only child, and his family history was unremarkable. Physical examination did not reveal specific findings for dyslipidemia such as xanthomas and hepatosplenomegaly. His vital signs were stable with mild fever and mild tachycardia (blood pressure, 85/45 $\mathrm{mmHg}$; heart rate, 140 beats/min; respiratory rate, 35 breaths/min; body temperature, $37.8^{\circ} \mathrm{C}$ ). Laboratory investigation revealed pronounced hypertriglyceridemia $(>5,000 \mathrm{mg} / \mathrm{dL}$; normal range, 50 to $150 \mathrm{mg} / \mathrm{dL}$ ), and hypercholesterolemia with total cholesterol at $988 \mathrm{mg} / \mathrm{dL}$ (normal range, 120 to $200 \mathrm{mg} / \mathrm{dL}$ ), high-density lipoprotein cholesterol at $390 \mathrm{mg} / \mathrm{dL}$ (normal range, 40 to $75 \mathrm{mg} / \mathrm{dL}$ ), and low-density lipoprotein cholesterol at $9 \mathrm{mg} / \mathrm{dL}$ (normal range, 70 to $160 \mathrm{mg} / \mathrm{dL}$ ). Amylase and lipase levels were normal. Serum albumin was slightly lower than normal ( $3.2 \mathrm{~g} / \mathrm{dL}$; normal range, 3.8 to $5.4 \mathrm{~g} / \mathrm{dL})$, but there was no proteinuria or clinical symptom. The analysis of lipoproteins showed high chylomicron at $24.0 \%$ (normal range, $0-0.4$ ), low alpha lipoprotein at $0.4 \%$ (normal range, 15.6-39.1), and low beta lipoprotein at $18.5 \%$ (normal range, 37.2-64.0). Primary chylomicronemia was diagnosed on this basis.

Table 1. Plasma exchange technical parameters in 1-month-old infant with chylomicronemia

\begin{tabular}{lc}
\hline $\begin{array}{l}\text { Technical parameters for } \\
\text { therapeutic plasma exchange }\end{array}$ & Value \\
\hline Patient's body weight $(\mathrm{kg})$ & 4.6 \\
Anticoagulation $(\mathrm{IU} / \mathrm{kg})$ & Heparin bolus, 30 \\
Blood flow rate $(\mathrm{mL} / \mathrm{min})$ & 6 \\
Estimated blood volume $(\mathrm{mL})$ & 345 \\
Removed volume $(\mathrm{mL})$ & \\
$\quad$ Output & 373 \\
Input & 318 \\
Total processing time (min) & 150 \\
\hline
\end{tabular}

The first therapeutic measure was to give a diet free of long chain fatty acids by means of MCTs milk. In addition, therapeutic plasmapheresis was necessary to manage the high risk of pancreatitis. Therapeutic plasma exchange (Spectra optia, Thermo BCT Inc., Lakewood, CO, USA) was carried out using fresh frozen plasma and $20 \%$ albumin. Body weight was 4.6 $\mathrm{kg}$ and the patient's estimated blood volume was $345 \mathrm{~mL}$. The blood flow rate was $6 \mathrm{~mL} / \mathrm{min}$ and total procedure time was 150 minutes (removed volume, $373 \mathrm{~mL}$; replaced volume, $318 \mathrm{~mL}$ ) (Table 1). Anticoagulation was also performed by heparin bolus (30 IU $/ \mathrm{kg}$ ). During the procedure, vital signs, bleeding tendency, and electrolytes were carefully monitored, and there were no significant side effects such as hypovolemic shock. The patient tolerated the procedure, and blood samples were collected before and 24, 48, and 72 hours after the procedure to assess the TG rebound. Immediately after plasmapheresis, the TG level had dropped to $2,125 \mathrm{mg} / \mathrm{dL}$ and then gradually decreased to $1,230 \mathrm{mg} / \mathrm{dL}, 465 \mathrm{mg} / \mathrm{dL}$, and $444 \mathrm{mg} / \mathrm{dL}$ after 24 hours, 48 hours, and 72 hours, respectively (Fig. 1). In addition, we started fibrate medication (Bezalip $30 \mathrm{mg} / \mathrm{kg}$ per day) and MCT oil. The patient was discharged 6 days after admission, and he continues to be regularly followed-up in the outpatient clinic to monitor his lipid profiles. For now, he is 1 year old with normal growth parameters; his height is $77 \mathrm{~cm}$ (25th-50th percentile) and weight is $10.2 \mathrm{~kg}$ (25th-50th percentile). The TG level stayed below $700 \mathrm{mg} / \mathrm{dL}$ during 1 year with same fibrate dosage without complications.

To identify the cause of chylomicronemia, genetic direct sequencing was performed. During the genetic investigation, a novel homozygous mutation was revealed in exon 4 of GPIHBP1 (c.476delG, p.Gly159Alafs), the gene that stabilizes the binding of chylomicrons near LPL and supports lipolysis (Fig. 2). Furthermore, genetic sequencing was performed for both parents, and his father harbored a heterozygous mutation, whereas, his mother had no mutation in GPIHBP1.

\section{Discussion}

In this study, we have described a 1-month-old infant who

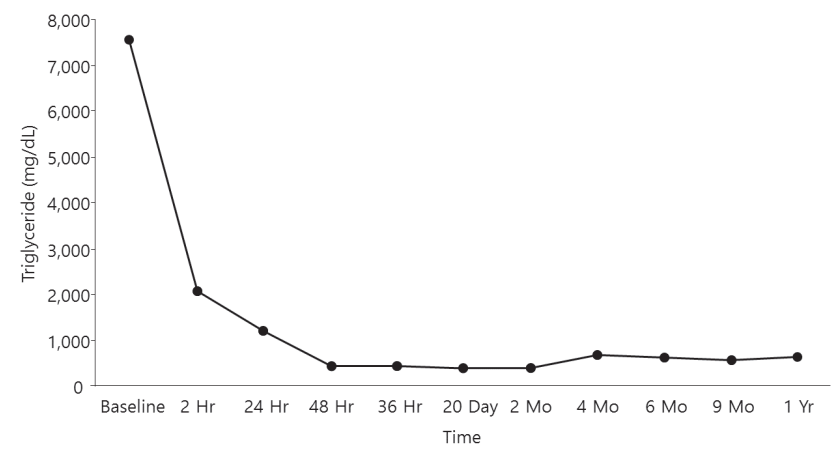

Fig. 1. Plasma triglyceride and cholesterol levels before and after plasma exchange. 
GPIHBP1 exon4

CAAGGGGGCAGGCGGCCCCCGGGCAGCTCCGAAACTGTGGGCGCAGCCC

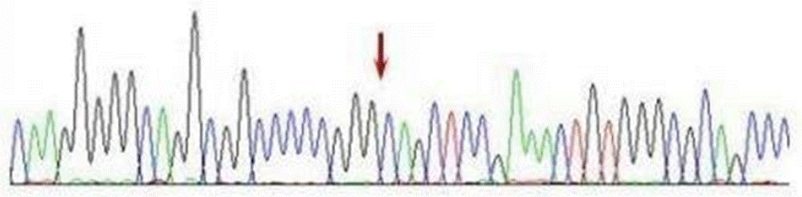

c.476de1G

Fig. 2. The mutation of nucleotide sequence in GPIHBP1 gene in patient

was incidentally found to have chylomicronemia resulting from a GPIHBP1 mutation and treated by plasma exchange. Patients with TG levels greater than $885 \mathrm{mg} / \mathrm{dL}$ should be investigated by a step-wise approach. Secondary causes such as uncontrolled diabetes, hypothyroidism, poor diet, alcohol use, nephrotic syndrome, obesity, and a variety of medications can be responsible for severe hypertriglyceridemia in children ${ }^{13)}$. If there is no secondary cause, monogenic factors should be considered. LPL deficiency is the most common cause of monogenic chylomicronemia. In the past, biochemical studies have been used to determine LPL or APOC2 activities, but nowadays genetic sequencing is performed for diagnosis. Patients with monogenic chylomicronemia show homozygosity or compound heterozygosity for loss-of-function mutations in causative genes such as $L P L, A P O C 2, A P O A 5, L M F 1$, and GPIHBP $1^{14)}$. Mutations in GPIHBP 1 , the most recently discovered gene, make up only $2 \%$ of monogenic mutations ${ }^{2}$. In the case at hand, the patient had a homozygous GPIHBP1 mutation and his father had a heterozygous mutation. Therefore, we can suppose that one allele was inherited from his father, and the other was from de novo mutation. Also, there was a possibility of paternal uniparental disomy as well. To our knowledge, only a few cases of infant monogenic chylomicronemia due to GPIHBP1 mutations have been reported. One was a 2-month-old infant homozygous for GPIHBP1 deletions who was treated with a low-fat formula $\operatorname{diet}^{8}$. The other was a 6-month-old infant with novel biallelic mutations in GPIHBP1 who improved on MCT-rich formula ${ }^{15)}$. The case at hand is therefore the youngest case with GPIHBP1 mutations who has been treated by plasmapheresis reported so far.

The mainstay of chylomicronemia therapy is an extremely low-fat diet, and especially in infants supplemental MCTs provide additional calories without raising circulating TG levels. However, the long-term effects of diets with high levels of MCTs remain unknown ${ }^{15}$. In addition, traditional lipid-lowering medications, such as fibrates, niacin and statins, are minimally effective in patients with primary chylomicronemia, especially patients with complete loss-of-function mutations in the lipolytic pathway ${ }^{2)}$. Although there are few studies about efficacy and safety of fibrates in children with hypercholesterolemia,
Becker et al. ${ }^{16)}$ reported fibrate is an alternative, safe and effective therapeutic choice for treatment of severe familial hypercholesterolemia in children. Therefore, we prescribed fibrate medication (Bezalip, $30 \mathrm{mg} / \mathrm{kg}$ per day) to reduce TG level definitely after single plasmapheresis.

Plasmapheresis or plasma exchange with direct removal of TG has been used, and plasma exchange is mentioned in the category III of the American Society for Apheresis 2010 guidelines (recommendation grade 2C, type of evidence II-I) for the treatment of hypertriglyceridemia ${ }^{17)}$. In infant patients, Stefanutti et al. ${ }^{18)}$ have reported successful plasmapheresis in a 25-day-old, 3.9-kg male with chylomicronemia whose TG level reached 38,000 mg/dL. Also Stefanutti et al. ${ }^{19)}$ reported a 3-month-old infant with chylomicronemia treated with plasma exchange to avoid acute pancreatitis, whose TG level dropped dramatically within 72 hours (Initial TG level was $167.00 \mathrm{mmol} / \mathrm{L}, 11.68 \mathrm{mmol} / \mathrm{L}$ after 72 hours after plasma exchange). In comparison with conservative management of chylomicronemia in a 2-month infant reported by Rios et al. ${ }^{8)}$ (Initial TG level was $>25,000 \mathrm{mg} / \mathrm{dL}, 397 \mathrm{mg} / \mathrm{dL}$ after 12 days), the use of plasma exchange showed effective removal of TG level. However, small clinical trials comparing plasmapheresis with conservative management have shown that plasmapheresis has no overall benefit ${ }^{4)}$. In addition, the TG concentration may return to the preapheresis values after plasmapheresis and there are no precise guidelines for therapeutic plasmapheresis in chylomicronemia. In our case, the TG level was severely raised, to above $5,000 \mathrm{mg} / \mathrm{dL}$, and we opted for a drastic management strategy, therapeutic plasmapheresis, to prevent the acute pancreatitis. Fortunately, this reduced the TG level dramatically without complications, to below $400 \mathrm{mg} / \mathrm{dL}$, and it stayed below $700 \mathrm{mg} / \mathrm{dL}$ for 1 year after only single plasmapheresis. Therefore, plasmapheresis may be an effective management strategy in infant patients with chylomicronemia, even though this is an invasive and risky procedure.

Recently, a new therapeutic approach has emerged with lomitapide (microsomal triglyceride transfer protein inhibitor). This drug is currently approved in North America and Europe for the treatment of homozygous familial hypercholesterolemia and has been shown to reduce triglyceride levels by $30 \%-40 \%{ }^{20)}$. However, lomitapide is only recommended for patients with pancreatitis associated with severe hypertriglyceridemia and without active hepatitis ${ }^{20)}$. In addition, the safety of this drug for infant patients has not been proven. Further clinical trials in younger children are needed.

In conclusion, we report the youngest case of chylomicronemia due to GPIHBP1 mutation that has been successfully treated by plasmapheresis. Accordingly, this approach may be effective in infant patients with severe chylomicronemia.

\section{Conflict of interest}

No potential conflict of interest relevant to this article was reported. 


\section{References}

1. Hegele RA, Ginsberg HN, Chapman MJ, Nordestgaard BG, Kuivenhoven JA, Averna M, et al. The polygenic nature of hypertriglyceridaemia: implications for definition, diagnosis, and management. Lancet Diabetes Endocrinol 2014;2:655-66.

2. Brahm AJ, Hegele RA. Chylomicronaemia: current diagnosis and future therapies. Nat Rev Endocrinol 2015; 11:35262.

3. Santamarina-Fojo S. The familial chylomicronemia syndrome. Endocrinol Metab Clin North Am 1998;27:55167.

4. Valdivielso P, Ramírez-Bueno A, Ewald N. Current knowledge of hypertriglyceridemic pancreatitis. Eur J Intern Med 2014;25:689-94.

5. Young SG, Zechner R. Biochemistry and pathophysiology of intravascular and intracellular lipolysis. Genes Dev 2013;27:459-84.

6. Johansen CT, Kathiresan S, Hegele RA. Genetic determinants of plasma triglycerides. J Lipid Res 2011;52:189-206.

7. Adeyo O, Goulbourne CN, Bensadoun A, Beigneux AP, Fong LG, Young SG. Glycosylphosphatidylinositolanchored high-density lipoprotein-binding protein 1 and the intravascular processing of triglyceride-rich lipoproteins. J Intern Med 2012;272:528-40.

8. Rios JJ, Shastry S, Jasso J, Hauser N, Garg A, Bensadoun A, et al. Deletion of GPIHBP1 causing severe chylomicronemia. J Inherit Metab Dis 2012;35:531-40.

9. Davies BS, Beigneux AP, Barnes RH 2nd, Tu Y, Gin P, Weinstein MM, et al. GPIHBP1 is responsible for the entry of lipoprotein lipase into capillaries. Cell Metab 2010;12:4252 .

10. Beigneux AP, Davies BS, Gin P, Weinstein MM, Farber E, Qiao X, et al. Glycosylphosphatidylinositol-anchored highdensity lipoprotein-binding protein 1 plays a critical role in the lipolytic processing of chylomicrons. Cell Metab 2007;5:279-91.
11. Patni N, Brothers J, Xing C, Garg A. Type 1 hyperlipoproteinemia in a child with large homozygous deletion encompassing GPIHBP1. J Clin Lipidol 2016;10:2016;9: 265-70.

12. Gotoda T, Shirai K, Ohta T, Kobayashi J, Yokoyama S, Oikawa $\mathrm{S}$, et al. Diagnosis and management of type I and type $\mathrm{V}$ hyperlipoproteinemia. J Atheroscler Thromb 2012;19:1-12.

13. Yuan G, Al-Shali KZ, Hegele RA. Hypertriglyceridemia: its etiology, effects and treatment. CMAJ 2007;176:1113-20.

14. Basel-Vanagaite L, Zevit N, Har Zahav A, Guo L, Parathath S, Pasmanik-Chor M, et al. Transient infantile hypertriglyceridemia, fatty liver, and hepatic fibrosis caused by mutated GPD1, encoding glycerol-3-phosphate dehydrogenase 1. Am J Hum Genet 2012;90:49-60.

15. Ahmad Z, Wilson DP. Familial chylomicronemia syndrome and response to medium-chain triglyceride therapy in an infant with novel mutations in GPIHBP1. J Clin Lipidol 2014;8:635-9.

16. Becker M, Staab D, Von Bergman K. Long-term treatment of severe familial hypercholesterolemia in children: effect of sitosterol and bezafibrate. Pediatrics 1992;89:138-42.

17. Szczepiorkowski ZM, Winters JL, Bandarenko N, Kim HC, Linenberger ML, Marques MB, et al. Guidelines on the use of therapeutic apheresis in clinical practice-evidence-based approach from the Apheresis Applications Committee of the American Society for Apheresis. J Clin Apher 2010;25:83-177.

18. Stefanutti C, Lanti A, Di Giacomo S, Mareri M, De Lorenzo F, Landolfo A, et al. Therapeutic apheresis in low weight patients: technical feasibility, tolerance, compliance, and risks. Transfus Apher Sci 2004;31:3-10.

19. Stefanutti C, Gozzer M, Pisciotta L, D'Eufemia P, Bosco G, Morozzi C, et al. A three month-old infant with severe hyperchylomicronemia: molecular diagnosis and extracorporeal treatment. Atheroscler Suppl 2013;14:73-6.

20. Sacks FM, Stanesa M, Hegele RA. Severe hypertriglyceridemia with pancreatitis: thirteen years' treatment with lomitapide. JAMA Intern Med 2014;174:443-7. 